\title{
Green in tooth and claw
}

\section{Demons in Eden: The Paradox of Plant \\ Diversity \\ by Jonathan Silvertown \\ University of Chicago Press: 2005.192 pp. \\ $\$ 25, £ 17.50$}

\section{Peter D. Moore}

Apart from a few parasites and decomposers, all plants face the same problem: how to capture light energy and fix atmospheric carbon dioxide more effectively than their neighbours. Actually, a darwinian would argue that their ultimate problem is how to leave more progeny than their neighbours, and that photosynthetic competition is just one aspect of this struggle. Growth may help, but pollination, seed maturation, fruit dispersal and seedling establishment all contribute to the final outcome in the next generation.

The theory of natural selection is based on the idea that heritable variation, acted on by the pressures of the environment, ultimately favours excellence. Only the fit survive. This being the case, one might predict that one supremely fit plant species, capable of photosynthesis, vegetative growth, pollination and seed dispersal in a way that outperformed all other plants, might ultimately prove the victor in the evolutionary struggle and would dominate the world's primary production. But this has not happened.

On the contrary, Earth displays an extra-

\section{EXHIBITION \\ In the croak room}

Nearly one-third of the world's amphibian species are endangered, and countless have already been lost. Frogs used to be creatures of the wild, but are fast becoming creatures of the lab - pickled, jarred and preserved as a static piece of history.

Now some long-dead frogs are taking centre stage as part of an exhibitionat the University of Kansas. Creatures from the university's herpetology collection, along with some from the Field Museum in Chicago, have been cast in urethane in a floor-to-ceiling display of froggy glory.

The show was created by Tracy Hicks, an artist from Texas who has been fascinated by natural history collections since childhood, when he hatchedeggs from turtles, lizards and snakes in his bedroom. The new installation is his tribute to vanishing species and to the science and art of collecting.

Floating in more than 1,300 jars are casts of some 79 species of frogs and tadpoles, many of themextinct, others endangered. They include Atelopus ignescens - once so common in the Andes that they would crunch frequently and ordinary diversity of plant species (around 400,000 ), almost all of which contribute to the planet's energy-trapping potential. Why? How can biodiversity be reconciled with natural selection? This is the question Jonathan Silvertown asks in Demons in Eden as he explores the remarkable variations of form and the elaborate systems of coevolution with animals that are found throughout the plant kingdom.

Among plants there are some darwinian demons that seem unstoppable in their spread, such as the kudzu vine, which seems intent on burying Florida, or the rhododendron, which is running amok in British woodlands. But both of these were introduced by humans, taken to parts of the world where there seem to be no constraints upon their spread. And herein seems to lie the answer. Constraints on population expansion decelerate the tendency for any plant species to spread indefinitely and become the ultimate demon. Such constraints include herbivores, seed predators, parasites, diseases and new evolutionary developments in competitor plants.

When a plant escapes from these constraints, it may for a while become a darwinian demon. For example, genetic evidence seems to show that the olive tree invaded the Canary Islands just once. Freed from predators and competitors it may well have spread unrestrained. There is still nothing on the Canaries that eats olive seeds, but the arrival of other tree species and the introduction of goats by people supplied the constraints that put the demon olive back in chains.

Another type of constraint on indefinite population expansion in plants is the supply of resources. Soil elements, such as nitrogen, phosphorus, potassium and calcium, are not always abundant, and the one that falls farthest behind the plant's demands may become the limiting factor for its growth. Silvertown describes the long-running Park Grass experiment at Rothamsted in England, and shows how the addition of an element, such as nitrogen, that may be limiting to some plant species, causes changes in the composition of grassland, releasing some species from the constraints of element limitation and causing others to be suppressed by the growth of the unchained demons.

The great diversity among plants does not therefore contradict darwinian principles, but upholds them. Vegetation illustrates the complex balance of interacting forces and trade-offs that evolution generates among competing species. In this highly readable and pleasantly anecdotal account of the dynamics of the plant world, Silvertown suggests that tasting the fruit of evolutionary knowledge may provide us with a ticket for readmission to the Garden of Eden, where we can exercise the privilege by ensuring that biodiversity is conserved. Let us hope that he is right.

Peter D. Moore is in the Division of Life Sciences,

King's College London, Franklin-Wilkins

Building, 150 Stamford Street,

London SE1 9NH, UK.

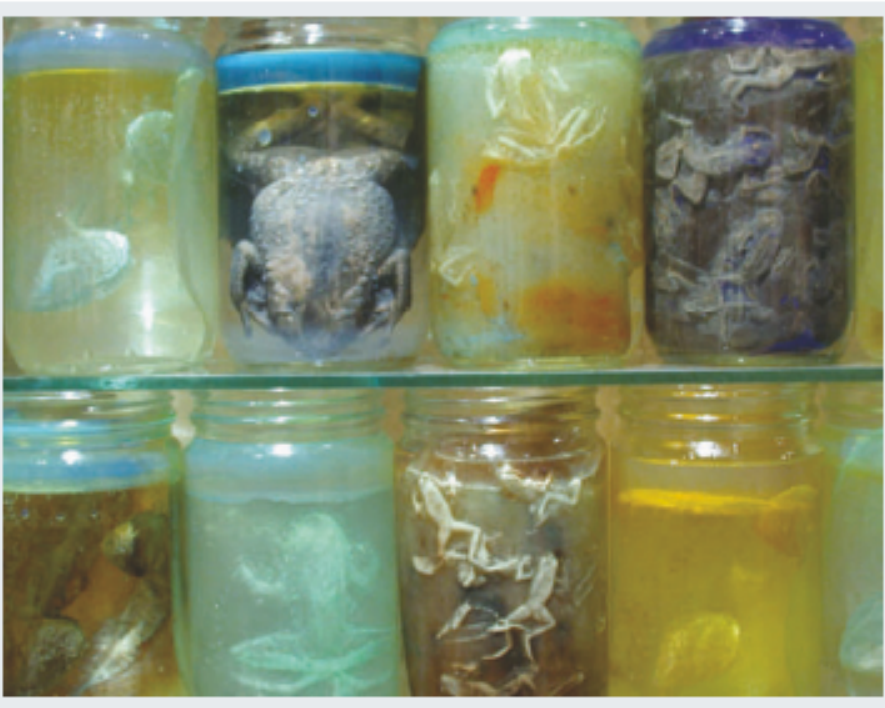

Kansas, eventually gave him permission to work with theirpriceless specimens. "This was an opportunity to show that the scientific collection has an aesthetic value to it, a certain beauty," says John Simmons, manager of the university's collection and a collaborator on the exhibit. Most strikingly, Hicks' translucent casts fluoresce

unpleasantly under the tyres of passing cars and the extinct golden toad of Costa Rica's cloud forests. Other featured species are just bizarre, such as the flying frogs from Asia or the paradox frog, in which a tadpole $25 \mathrm{~cm}$ long metamorphoses into an 8-cm frog.

In severalcases, Hicks made his casts from the species holotype. He practised for years on less valuable specimens to perfect his technique, and both museums, in Chicago and when an ultraviolet light is switched on transforming the wall of jars into an eerie spectacle, where details such as skin impressions pop out, glowing blue or green. The effect communicates the life of the animals. Audio recordings add to the impression, with a frog chorus of now-vanished species echoing their chants along the walk.

The exhibit, 'Two Cultures:Collection', will remain on display until March $2006 . \quad$ A.W. 